\title{
Disponibilidade de irmãos no Brasil: um estudo metodológico sobre relações de parentesco
}

\author{
Francismara Fernandes Guerra* \\ Simone Wajnman ${ }^{\star \star}$ \\ Cássio M. Turra ${ }^{\star \star \star}$
}

Quando a fecundidade declina, não é somente o número de filhos que se torna menor, mas também o número de irmãos. Para aferir as mudanças ocorridas sobre a disponibilidade de irmãos no Brasil, o presente estudo emprega um método que se destina a estimar o número esperado de irmãos nascidos vivos e sobreviventes, por meio de modelos matemáticos que utilizam apenas taxas de fecundidade e de mortalidade. Os resultados indicam que, no início da transição demográfica brasileira, a média de irmãos nascidos vivos se estabelece em patamares elevados e sofre um forte declínio durante o processo, motivado principalmente pela queda da fecundidade. Observa-se, ainda, que a média de irmãos sobreviventes nas idades mais avançadas tende a ser muito semelhante para coortes mais velhas e mais novas, mas o número de irmãos sobreviventes durante a infância dessas coortes tende a diferenciar-se fortemente. Isso ocorre porque, por um lado, o efeito da elevada mortalidade, principalmente a infantil, reduz a média de irmãos gerados pela alta fecundidade para as coortes mais velhas e, por outro, a queda da fecundidade diminui o número de irmãos nascidos vivos das coortes mais novas, ao mesmo tempo que a redução da mortalidade garante sua quase constância no ciclo de vida. Como conclusão, indica-se que: o número médio de irmãos nascidos vivos tende a se estabelecer em baixos níveis nos próximos anos; o número médio de irmãos sobreviventes tende a ser cada vez mais próximo da média do número de irmãos nascidos vivos; e, apesar dos baixos níveis de fecundidade corrente, não se pode falar em uma tendência de extinção dos irmãos e, por consequência, primos, tios, etc.

Palavras-chave: Transição demográfica. Modelo de estimativa das relações de parentesco. Disponibilidade de irmãos.

\footnotetext{
* Departamento de Economia Doméstica da Universidade Federal de Viçosa (UFV), Viçosa-MG, Brasil (francismarafernandes@ yahoo.com.br).

${ }^{\star *}$ Centro de Desenvolvimento e Planejamento Regional (Cedeplar), Universidade Federal de Minas Gerais (UFMG), Belo Horizonte-MG, Brasil (wajnman@cedeplar.ufmg.br).

${ }^{\star \star \star}$ Centro de Desenvolvimento e Planejamento Regional (Cedeplar), Universidade Federal de Minas Gerais (UFMG), Belo Horizonte-MG, Brasil (turra@cedeplar.ufmg.br).
} 


\section{Introdução}

Estudos demográficos recentes mostram que, quando a fecundidade declina, não é somente o número de filhos que se torna menor, mas também o de irmãos e, consequentemente, o número dos demais parentes colaterais, tal como tios, primos, etc. (MURPHY, 2010). Essa mudança estrutural da família suscita a preocupação de estudos que analisem a família em termos de tamanho e existência dos vários tipos de parentesco, considerando a transição da fecundidade. A relação de irmãos, especificamente, mostra-se de grande importância no processo de mudanças na estrutura da família, já que eles são os responsáveis diretos pela dinâmica do ciclo familiar colateral. De fato, níveis cada vez mais baixos de fecundidade sugerem um cenário de verticalização da família (MURPHY, 2009), no qual a extinção de parentesco colateral seria o resultado de uma situação extrema: a inexistência de irmãos.

Pesquisas sobre família que abordam a existência de irmãos, ainda que marginalmente sob o aspecto demográfico (correlação com a fecundidade e o ciclo de vida - casamento, morte, envelhecimento, etc.), mostram uma visão utilitarista de ter um irmão. Nesse sentido, a importância atribuída aos irmãos é significativamente determinante na decisão de ter filhos. Em termos gerais, é possível dizer que essa decisão está sujeita a basicamente três efeitos. 0 primeiro refere-se ao efeito do comprometimento, o qual trata a visão comum de que um casal deveria ter filhos ou de que uma família é incompleta sem filhos. O segundo corresponde ao efeito da paternidade e da maternidade, no qual se destaca a importância de ter filho para se afirmar como adulto perante a sociedade. E o terceiro trata-se do efeito da irmandade, que sugere haver a intenção de prover um irmão ao filho único da família (GRIFFITH et al., 1985; VIKAT et al., 1999; THOMSON, 1997). Este último efeito, o da irmandade, desloca a visão da família para a perspectiva dos filhos e tem sido pouco discutido na literatura demográfica em geral.

Nessa mesma linha de pensamento, pode-se ainda dizer que existem benefícios a serem compartilhados pela família que pesam na decisão de ter filhos sob a ótica da provisão de irmãos. Pelo primeiro filho tido, a família ganharia o valor do comprometimento, o homem ganharia o valor da paternidade e a mulher, por sua vez, o valor da maternidade. Pelo segundo filho tido, o ganho seria relativo ao valor do irmão. Já para nascimentos de maior ordem, não haveria maiores ganhos, ao menos na sociedade moderna, mas somente aumento no custo da criação dos filhos (THOMSON, 2004). Isso sugere que, em virtude da restrição orçamentária familiar, o número de irmãos deveria ser reduzido e definiria o tamanho da família.

Em outros termos, pode-se dizer que, à medida que a família cresce, cada filho passa a gozar uma proporção cada vez menor dos recursos financeiros destinados à educação e das transferências intergeracionais financeiras. Do mesmo modo, os recursos não materiais, como tempo, energia e educação informal, que os pais dedicam aos seus filhos seriam mais diluídos em famílias numerosas. Consequentemente, o tamanho da família 
alteraria não somente os recursos disponíveis para os filhos, mas também a trajetória de acumulação de riqueza, já que educação e transferências financeiras diretas moldam o processo decisório da acumulação de riqueza. Dessa forma, indivíduos pertencentes a grandes famílias tendem a acumular menos riqueza como resultado da divisão e recursos entre os irmãos, durante a infância (KEISTER, 2003).

Apesar das implicações negativas de se ter (muitos) irmãos, por outro lado, irmãos tendem a ser membros permanentes das redes sociais. Não são substitutos perfeitos de pais, cônjuges e filhos, mas podem ser preferíveis a estes na realização de determinadas tarefas. As relações entre irmãos podem ser instáveis, estando sujeitas aos efeitos do ciclo de vida. De modo geral, os contatos e trocas entre irmãos crescem com a dissolução marital e diminuem em virtude do nascimento de filhos, de novos casamentos e com a distância geográfica. A despeito de quaisquer evidências negativas, irmãos parecem ganhar grande importância com a idade, já que dividem suas histórias e reafirmam seu passado juntos (WHITE, 2001) e tendem a exercer papel fundamental no momento de dividir responsabilidades e compartilhar os cuidados com os pais idosos e, mais adiante, na formação de rede de suporte e cuidados com eles próprios na velhice (WOLF; FREEDMAN; SOLDO, 1997; ERIKSEN; GERSTEL, 2002; BIGBY, 2008).

Discutir e estudar a importância dos irmãos no ciclo de vida individual torna-se de grande valia social se as tendências demográficas indicarem a extinção ou uma redução significativa dos irmãos. Por isso, faz-se necessário, antes de tudo, compreender como a dinâmica da oferta dos irmãos se comporta na transição demográfica e quais são as tendências que podem ser identificadas nesse processo. Assim, este trabalho busca examinar, para o contexto brasileiro, a forma e a intensidade com que a transição demográfica afeta a evolução do número médio de irmãos. Especificamente, identificam-se para coortes de 1930 a 2010: a evolução do número médio de irmãos de ambos os sexos nascidos vivos e sobreviventes nas diversas idades ao longo do ciclo de vida; a evolução da proporção de irmãs sobreviventes no total de irmãos de ambos os sexos, nas distintas idades; e a proporção de irmãos mais novos entre o total de irmãos de ambos os sexos, nas distintas idades.

$\mathrm{Na}$ literatura brasileira, particularmente, os estudos das relações de parentesco se restringem, muitas vezes, à análise do tamanho e dos tipos de arranjos domiciliares. Isso porque, nas pesquisas domiciliares oficiais, o grau de parentesco das pessoas do domicílio é estabelecido sempre em relação ao chefe ou à pessoa de referência do domicílio da família, o que permite a investigação sobre os familiares conviventes no domicílio e, em geral, a identificação do parentesco entre as pessoas de cada família por dedução. Mas, a partir de tais pesquisas, não se pode determinar a existência de parentes fora dos limites domiciliares. Por esse motivo, destaca-se a necessidade do emprego de métodos quantitativos que possibilitem a estimação das relações de parentescos, independentemente de tais informações. Na demografia, o trabalho desenvolvido por Goodman, Keyfitz e Pullum (1974), doravante denominados GKP, ganha destaque ao tratar das relações de parentesco por meio de modelos matemáticos, empregando apenas funções de fecundidade e de 
mortalidade. Seus métodos propõem, como é de nosso interesse, a estimativa da disponibilidade (ou número médio) de irmãos vivos e sobreviventes de um indivíduo aleatório (identificado como ego) em qualquer idade. No entanto, uma das limitações frequentemente apontadas para esse método é a adoção do pressuposto de que a população seja estável, o que implica sérias distorções nas estimativas em um contexto de instabilidade. Por essa razão, adaptou-se o método original de modo a incorporar funções de fecundidade e de mortalidade específicas para as distintas coortes analisadas.

\section{Antecedentes}

Keyftiz (1986) mostra que, ao longo do ciclo de vida, o número médio de irmãos mais velhos nascidos vivos que uma pessoa teria é constante para todas as idades. De fato, uma vez nascido, não é possível adquirir irmãos mais velhos. Por outro lado, o número de irmãos mais novos nascidos vivos tende a crescer assintoticamente até, aproximadamente, os 25 anos, a partir de quando se mantém constante. Isso ocorre porque dificilmente se adquirem novos irmãos da mesma mãe após essa idade. 0 autor mostra ainda que, como se espera, à medida que se envelhece, o número de irmãos sobreviventes, tanto mais velhos quanto mais novos, tende a cair.

Com a transição demográfica, Murphy (2010) verifica que o número de irmãos sobreviventes nas idades mais avançadas é muito semelhante para coortes mais velhas e mais novas. No entanto, o número de irmãos sobreviventes durante a infância dessas coortes é bastante distinto porque, por um lado, o efeito da elevada mortalidade, principalmente a infantil, reduz a média de irmãos gerados pela alta fecundidade para as coortes mais velhas e, por outro, a queda da fecundidade diminui o número de irmãos nascidos vivos das coortes mais novas, ao mesmo tempo que a redução da mortalidade garante a sobrevivência dos irmãos no final da vida dessas coortes. Os baixos níveis da fecundidade moderna resultam não somente em poucos irmãos na família nuclear, mas também em poucos parentes de todos os graus, o que implica um decréscimo do tamanho médio da família estendida, em decorrência do reduzido número de tios, sobrinhos, primos e outros parentes (KEYFTIZ, 1986).

No caso de diferentes regimes populacionais, Keyftiz (1986) observou que, em uma população estável com taxa de crescimento positiva, os irmãos mais novos são em maior número do que os mais velhos; em uma população estável com taxa de crescimento negativa, o cenário se inverte; e, em uma população estacionária, há tantos irmãos mais novos quanto mais velhos. Visto de outro modo, quando o crescimento populacional é positivo, a população é mais jovem, havendo, portanto, mais mulheres em idade reprodutiva e, consequentemente, mais filhos nascendo, os quais se agregarão ao grupo dos irmãos mais novos.

Em vista da forte relação existente entre crescimento populacional e número de irmãos, Goldman (1978) demonstra que se pode calcular a taxa intrínseca de crescimento populacional com base no número médio de irmãos mais novos e mais velhos. A autora conclui que 
esta é uma poderosa técnica a ser usada na ausência de dados vitais, mesmo que o método exija informações demográficas sobre a existência de irmãos mais velhos e mais novos.

Conforme Wachter (1980), a técnica proposta por Goldman (1978) destina-se ao estudo demográfico de comunidades relativamente pequenas, onde o recenseamento dos parentes é factível. No entanto, a teoria da população estável, utilizada para desenvolver a aproximação alternativa para a taxa de crescimento, ignora toda variabilidade aleatória nas taxas vitais, gerando ferramentas de estimação de parentesco pouco satisfatórias em cenários de grande instabilidade demográfica.

McDaniel e Hammel (1984), inspirados por Goldman (1978), propõem uma nova medida alternativa da taxa intrínseca de crescimento baseada nas medidas de parentescos, especificamente no número esperado de irmãos mais velhos (decanos) e no número esperado de irmãos mais novos (caçulas) entre todos os irmãos. As novas relações dependem não somente da oferta de determinado tipo de parentesco, mas também de uma medida temporal de reprodução. A medida apresentada por McDaniel e Hammel (1984) resulta da diferença entre a idade média dos irmãos mais velhos (decanos) e a idade média dos irmãos mais novos (caçulas) entre todos, enquanto a medida de Goldman (1978) corresponde à diferença média entre as idades de pares de irmãos. Para McDaniel e Hammel (1984), sua nova medida é mais robusta, pois é mais simples e está menos sujeita a variância amostral e viés por má-declaração.

Mudanças no regime demográfico, que causaram o envelhecimento da população, também provocaram o envelhecimento das relações geracionais. Segundo Murphy (2009), eventos que, tipicamente, ocorriam precocemente no ciclo de vida, como a morte dos avós e dos pais, assim como ter filhos e netos, passaram a ser postergados, o que resulta em diferentes transformações nas relações de parentesco. 0 autor mostra que a oferta de parentes ascendentes, como ter mãe viva, é mais suscetível às mudanças ocorridas na mortalidade, ao passo que a oferta dos demais tipos de parentes, como o número de irmãos, é mais suscetível à instabilidade da fecundidade. Por exemplo, em função da grande proporção de famílias que optam pelo padrão familiar de filho único, o peso de zero irmãos é potencializado pela presença dessas famílias e afeta fortemente a média de irmãos para baixo, conforme esclarece Murphy (2009). Isso, evidentemente, impacta de forma semelhante os níveis de parentescos colaterais, como tios, primos e sobrinhos. Por efeito das transformações familiares decorrentes da transição demográfica, a estrutura de parentesco caminha para a verticalização da família, ou para a família pé-de-feijão (bean-pole family), em que o número de parentes colaterais é reduzido.

Hammel (2005a) analisa a relação da dinâmica demográfica e as relações de parentesco sob o aspecto de choques populacionais por meio de microssimulação. 0 autor conclui que, em uma população estacionária, como esperado, não há mudanças significativas nos padrões de parentesco. Já quando há choques que produzem taxas de crescimento positivas, como queda na mortalidade e/ou aumento da fecundidade, o número de parentes aumenta. Para impactos que provocam taxas de crescimento negativas, como elevação da 
mortalidade e/ou queda da fecundidade, o efeito é contrário, isto é, o número esperado de parentes cai. Quando se aplicam choques alternados na fecundidade, são produzidos padrões complexos de serem descritos, sendo que resposta inicial é semelhante ao choque simples, contudo, com o tempo, esta resposta é modificada pelos subsequentes choques alternados. Enfim, os padrões de parentesco e a desigualdade na distribuição de parentes replicam os choques na fecundidade e a periodicidade das mudanças na estrutura etária.

Hammel (2005b) mostra que, com as mudanças demográficas, os grupos de parentes se alteram em tamanho e com relativa desigualdade. Os resultados obtidos pelo autor, a partir de um modelo analítico de parentesco com abordagem microeconômica, permitem generalizar a relação existente entre a distribuição de parentes e o crescimento populacional. Quando se eleva a taxa de crescimento populacional, o número de parentes cresce e a variabilidade do tamanho dos grupos familiares tende a diminuir; e, quando a taxa de crescimento populacional cai, o número de parentes se reduz e a variabilidade do tamanho dos grupos familiares tende a aumentar.

Além da oferta de parentes, em termos de média, e da sua relação com a dinâmica demográfica, as relações de parentesco também ganham atenção no tocante à frequência de parentes na população. Baseando-se na média e na variância da distribuição das parturições completas, Pullum (1982) desenvolve funções de probabilidade que descrevem a existência ou não de vários tipos de parentesco, encontrando resultados esperados para o contexto demográfico atual. Em um cenário plausível da transição demográfica para a estabilidade, o autor conjectura a existência de nenhum ou de poucos irmãos, tios, primos, sobrinhos ou netos para considerável parcela da população, demonstrando, por isso, a necessidade de maior aprofundamento na análise da dinâmica da disponibilidade de parentes na população e do levantamento de suas tendências, sobretudo no contexto da transição demográfica.

\section{Dados}

Para estimar a disponibilidade de irmãs e irmãos por coortes de nascimento compreendidas entre 1930 e 2010, no presente estudo foram utilizadas as séries de dados construídas por Wajnman (2012). Em suas estimativas da oferta de parentes feitas para 1960, 1970, 1980, 1990, 2000, 2010, a autora reconstrói funções de fecundidade e de mortalidade por coortes, desde 1875 até 2010.

Para as funções de taxas específicas de fecundidade por idade, foram combinadas duas fontes de dados. A primeira corresponde às funções de fecundidade apresentadas por Horta, Carvalho e Frias (2000), para coortes de mulheres que entraram no período reprodutivo entre 1905 e 1980. A segunda refere-se às funções de fecundidade por período quinquenal utilizadas pelo Instituto Brasileiro de Geografia e Estatística (IBGE) na atualização de 2008 para as projeções populacionais. Como essas últimas eram estimativas por períodos, foi necessário recompor funções de taxas específicas por coortes de mulheres entrando no 
período reprodutivo entre 1980 e 2010. Para o período entre 1875 e 1905, assumiu-se que as funções mantiveram-se constantes e iguais à função de 1905.

Para a estimativa da distribuição de nascimentos das filhas segundo a idade das mulheres, empregaram-se as taxas específicas de fecundidade para cada período e as estimativas de população do IBGE, por idade.

Para as funções de mortalidade, na ausência de uma série histórica de óbitos por coorte, optou-se por construir, primeiro, funções de período, que serviram de base para a recomposição das funções de coorte. Para esse propósito, tomou-se, inicialmente, o nível geral de mortalidade por ano-calendário, a cada cinco anos, a partir das informações do IBGE sobre a expectativa de vida ao nascer por sexo no Brasil, disponíveis para vários anos entre 1910 e 2010. Na ausência da informação para um ano específico, a expectativa de vida foi estimada por interpolação linear. Para os anos anteriores a 1910, assumiu-se que a expectativa de vida ao nascer manteve-se constante e igual à expectativa de vida ao nascer em 1910. 0 segundo passo foi construir as funções de mortalidade por idade, para cada ano-calendário. Optou-se, assim, pela solução simplificadora de adotar as funções por idade de uma mesma família de tabelas-modelo, para todo o período de análise. Foi escolhido o modelo Oeste de Coale e Demeny, em função do seu maior grau de generalidade. Assim, as funções de mortalidade por idade da família Oeste de Coale e Demeny foram interpoladas de tal forma que correspondessem às expectativas de vida ao nascer fornecidas pelo IBGE para cada ano. Dado que a idade máxima das tabelas modelo é 80 anos, utilizou-se um modelo logístico para estimar as probabilidades de mortalidade entre as idades de 80 e 130 anos (PRESTON; HEUVELINE; GUILLOT, 2001). Finalmente, construída a série histórica de funções de mortalidade por idade, sexo e ano, foi possível reconstituir as funções de coorte.

As séries de dados demográficos empregadas neste estudo estão estimadas, portanto, para cada cinco anos do período compreendido entre 1880 e 2010 e por grupos etários quinquenais. Os dados de mortalidade descrevem a sobrevivência até o limite de 115 anos, ao passo que o período reprodutivo das mulheres limita-se às idades entre 15 e 49 anos. Esta restrição pode incorrer no erro de subestimar o número de filhos, já que não incorpora a fecundidade precoce que ocorre entre mulheres de 10 a 14 anos, bem como a fecundidade tardia para aquelas de 50 a 54 anos. Acreditamos, no entanto, que isso não influencie significativamente as médias ou mude as tendências observadas nas estimativas de irmãs e irmãos.

A fim de gerar estimativas para grupos etários unitários ou por idade simples, interpolou-se a fecundidade acumulada dentro de cada grupo etário por meio dos multiplicadores de Grabill. Da mesma forma, foi realizada a redistribuição das mulheres que tiveram filhos por idade simples. Como a soma total das estimativas não se conservou, a diferença foi então redistribuída proporcionalmente em cada idade. Já a sobrevivência foi recalculada por idade simples, partindo do pressuposto implícito da tábua de vida de que os óbitos ocorridos em cada intervalo etário se distribuem uniformemente no mesmo intervalo. 
Assumiu-se, finalmente, que as coortes cujo ano de nascimento pertença ao mesmo quinquênio estão submetidas às mesmas funções de maternidade e sobrevivência, uma vez que os dados originais são estimados para grupos quinquenais.

\section{Método}

Os modelos matemáticos desenvolvidos por Goodman, Keyfitz e Pullum (1974) ganharam especial atenção na demografia não somente por permitirem a estimação da oferta de diversos parentescos, empregando apenas as funções de fecundidade e de mortalidade tradicionalmente conhecidas, mas também por suas limitações e pressupostos. A restrição dos modelos de apenas um sexo e a suposição de estabilidade demográfica assumida nos cálculos são flexibilizadas neste artigo, configurando-se como um avanço em relação às técnicas originais, embora o pressuposto de homogeneidade das taxas vitais seja ainda mantido.

Ao se modelar a estimação dos irmãos (sexo masculino) e incorporar as estimativas das irmãs (sexo feminino), ego passa a ser uma pessoa média que pode assumir qualquer um dos sexos. Assim, sem a necessidade de qualquer formulação matemática, ego passa a ser definido como a pessoa de referência para quem se estima o número esperado de parentes (neste caso, irmãs e irmãos), na condição de que ego seja uma mulher viva ou um homem vivo escolhida(o) aleatoriamente.

Em nossa modelagem, propomos aproximações discretas das fórmulas matemáticas para a estimação da disponibilidade de irmãs propostas por GKP, em que foram utilizadas funções definidas para grupos etários unitários. A lógica empregada segue o seguinte raciocínio: os irmãos e as irmãs de ego são os filhos e as filhas da possível mãe de ego. Uma vez que não se conhece a idade da mãe de ego, também se faz necessário calcular o número de filhos e filhas que sua possível mãe poderia ter tido antes e depois de seu nascimento. Por conseguinte, para estimar o número total de irmãos e irmãs de ego, é necessário calcular separadamente seus irmãos e irmãs mais novos e seus irmãos e irmãs mais velhos. Dessa forma, o número esperado de irmãos nascidos vivos e o número esperado de irmãs nascidas vivas, ambos mais velhos, que ego teria, na idade simples no tempo $t$, dado que sua mãe tinha $x$ anos ao seu nascimento e $y$ anos ao nascimento de seus irmãos, são respectivamente:

$\sum_{x=\alpha+1}^{x=\beta}\left[\sum_{y=\alpha}^{y=x-1} F_{y}^{M}(t-a-x+y)\right]_{1} W_{x}(t-a)$

$\sum_{x=\alpha+1}^{x=\beta}\left[\sum_{y=\alpha}^{y=x-1} F_{y}^{F}(t-a-x+y)\right]_{1} W_{x}(t-a)$

Onde: $\alpha$ e $\beta$ são a idade inicial e a idade final, respectivamente, do período reprodutivo feminino; ${ }_{1} F_{y}^{M}$ e ${ }_{1} F_{y}^{F}$ são as taxas específicas de fecundidade por idade simples de nascimentos masculinos e femininos, respectivamente, observadas no tempo $t-a-x+y$; e ${ }_{1} W_{x}$ é a distribuição etária por idade simples das mulheres que tiveram filhas ou filhos nascidos vivos na população no momento do nascimento de ego $t$ - $a$. 
Observe que o número de irmãs e irmãos mais velhos nascidos vivos continua não dependendo da idade de ego, como nas fórmulas originais. 0 que depende da idade de ego, na verdade, é o ano de nascimento do(a) irmão(ã) de ego $t-a-x+y$, já que estamos tratando de diferentes coortes de nascimento.

O número esperado de irmãos sobreviventes e o número esperado de irmãs sobreviventes, ambos mais velhos, que ego teria na idade $a$, no tempo $t$, são respectivamente:

$\sum_{x=\alpha+1}^{x=\beta}\left[\sum_{y=\alpha}^{y=x-1}{ }_{1} F_{y}^{M}(t-a-x+y){ }_{1} L_{a+x-y}^{M}(t)\right]_{1} W_{x}(t-a)$

$\sum_{x=\alpha+1}^{x=\beta}\left[\sum_{y=\alpha}^{y=x-1}{ }_{1} F_{y}^{M}(t-a-x+y){ }_{1} L_{a+x-y}^{F}(t)\right]_{1} W_{x}(t-a)$

Onde: $L_{a+x-y}^{M} \mathrm{e}_{1} L_{a+x-y}^{F}$ são as proporções de sobreviventes dos sexos masculino e feminino, respectivamente, observadas no tempo $t$ da coorte de nascimento, dos irmãos e das irmãs, em $t-a-x+y$.

Note-se que o número de irmãos mais velhos (incluindo as irmãs) - nascidos vivos ou sobreviventes - é sempre zero se a idade da mãe de ego ao seu nascimento é a idade inicial do período reprodutivo feminino $(x=\alpha)$. De fato, mulheres que acabaram de entrar no período reprodutivo não podem gerar irmãs e irmãos mais velhos. Também mulheres com a idade final do período reprodutivo feminino $(x=\beta)$ não podem gerar irmãs e irmãos mais velhos, porque ego não poderia nascer depois do fim do período reprodutivo feminino.

Já para o cálculo dos irmãos e irmãs mais novos de ego, introduz-se a condição (razão) de sobrevivência de sua mãe após seu nascimento até o nascimento de seu irmão. Assim, o número esperado de irmãos nascidos vivos e o número esperado de irmãs nascidas vivas, ambos mais novos, que ego teria na idade $a$ no tempo $t$, são respectivamente:

$$
\begin{aligned}
& \sum_{x=\alpha}^{x=\beta-1}\left[\sum_{y=x+1}^{y=x+a} \frac{{ }_{1} L_{y}^{F}(t-a-x+y)}{{ }_{1} L_{x}^{F}(t-a)}{ }_{1} F_{y}^{M}(t-a-x+y)\right]{ }_{1} W_{x}(t-a) \\
& \sum_{x=\alpha}^{x=\beta-1}\left[\sum_{y=x+1}^{y=x+a} \frac{{ }_{1} L_{y}^{F}(t-a-x+y)}{{ }_{1} L_{x}^{F}(t-a)}{ }_{1} F_{y}^{F}(t-a-x+y)\right]{ }_{1} W_{x}(t-a)
\end{aligned}
$$

Onde: ${ }_{1} L_{y}^{F} /{ }_{1} L_{x}^{F}$ corresponde à chance de sobrevivência das possíveis mães de ego a contar do nascimento de ego até o nascimento de seu irmão ou de sua irmã (entre as idades $x$ e $y$ e entre os períodos $t-a$ e $t-a-x+y)$.

Por fim, o número esperado de irmãos sobreviventes e o número esperado de irmãs sobreviventes, ambos mais novos, que ego teria na idade $a$ no tempo $t$, são respectivamente:

$$
\begin{gathered}
\sum_{x=\alpha}^{x=\beta-1}\left[\sum_{y=x+1}^{y=x+a} \frac{{ }_{1}^{F} L_{y}^{F}(t-a-x+y)}{{ }_{1} L_{x}^{F}(t-a)}{ }_{1} F_{y}^{M}(t-a-x+y){ }_{1} L_{a+x-y}^{M}(t)\right]{ }_{1} W_{x}(t-a) \\
\sum_{x=\alpha}^{x=\beta-1}\left[\sum_{y=x+1}^{y=x+a} \frac{{ }_{1} L_{y}^{F}(t-a-x+y)}{{ }_{1} L_{x}^{F}(t-a)}{ }_{1} F_{y}^{F}(t-a-x+y){ }_{1} L_{a+x-y}^{F}(t)\right]{ }_{1} W_{x}(t-a)
\end{gathered}
$$


Observe-se, ainda, que os nascimentos das irmãs e dos irmãos mais velhos de ego não são condicionados à sobrevivência de sua mãe, dado que, para ego nascer, sua mãe tem que estar viva.

O número de irmãs e irmãos mais novos - nascidos vivos ou sobreviventes - é sempre zero quando ego é recém-nascido(a), isto é, recém-nascidos podem ter somente irmãs e irmãos mais velhos. Além disso, o número de irmãs e irmãos mais novos (nascidos vivos ou sobreviventes) é sempre zero se a idade da mãe de ego ao seu nascimento é a idade final do período reprodutivo feminino $(x=\alpha)$, dado que mulheres que encerraram o período reprodutivo não podem gerar mais filhos(as). Analogamente, mulheres ao início do período reprodutivo $(x=\beta)$ não geram irmãs e irmãos mais novos, pois ego nasce previamente a seus irmãos e irmãs.

\section{Análise dos resultados}

O Gráfico 1 apresenta as estimativas do número de irmãs e irmãos nascidos vivos e sobreviventes, para o período de 1930 a 2010, segundo a idade de ego. No ciclo de vida, o número médio de irmãs e irmãos nascidos vivos cresce assintoticamente com a idade até, aproximadamente, os 20 anos, mas se mantém realmente constante após os 35 anos de idade. Ao mesmo tempo, o número de irmãs e irmãos sobreviventes aumenta rapidamente também até, aproximadamente, os 20 anos, começando a declinar a partir dessa idade.

É interessante notar que, com a transição demográfica, o número médio de irmãs e irmãos nascidos vivos apresenta decréscimos, cada vez mais acentuados, à medida que as coortes são mais jovens. Por outro lado, as estimativas do número médio de irmãs e irmãos sobreviventes para as coortes mais jovens tendem a superar, com a evolução da idade, as estimativas das coortes mais velhas. Por exemplo, um(a) recém-nascido(a) da coorte de 1930 conta com 2,9 irmãs e/ou irmãos nascidos vivos anteriormente a seu nascimento e, aos 35 anos de idade, com 4,0 irmãs e/ou irmãos sobreviventes. Já para um membro da coorte de 1970, um indivíduo médio nasce após 2,4 irmãs e/ou irmãos nascidos vivos e conta com 4,6 irmãs e/ou irmãos sobreviventes aos 35 anos de idade. Ou seja, para as coortes anteriores a 1970, o efeito da redução da mortalidade se sobrepõe ao da queda da fecundidade - e da consequente diminuição do número de irmãos nascidos vivos -, produzindo um número cada vez maior de irmãos sobreviventes. Esse é um achado importante que indica que, ao contrário do que sugerem as tendências associadas à redução do tamanho das famílias e à sua maior verticalização, os idosos atuais e das próximas duas décadas tendem a ter um número médio de irmãos e irmãs sobreviventes maior do que o que tinham os idosos das coortes anteriores na mesma idade. Isso corrobora a noção de que, do ponto de vista da oferta de parentes, que cria a rede potencial de suporte para os idosos, a queda da mortalidade implica um "bônus" em termos da disponibilidade de irmãos sobreviventes. 
GRÁFICO 1

Número total de irmãs e irmãos nascidos vivos e sobreviventes, por coorte de nascimento de ego, segundo a idade de ego

Brasil - 1930-2010

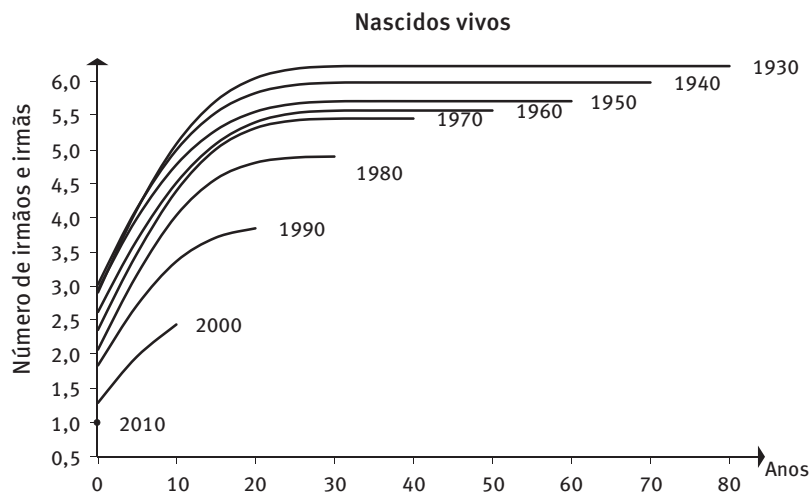

Sobreviventes

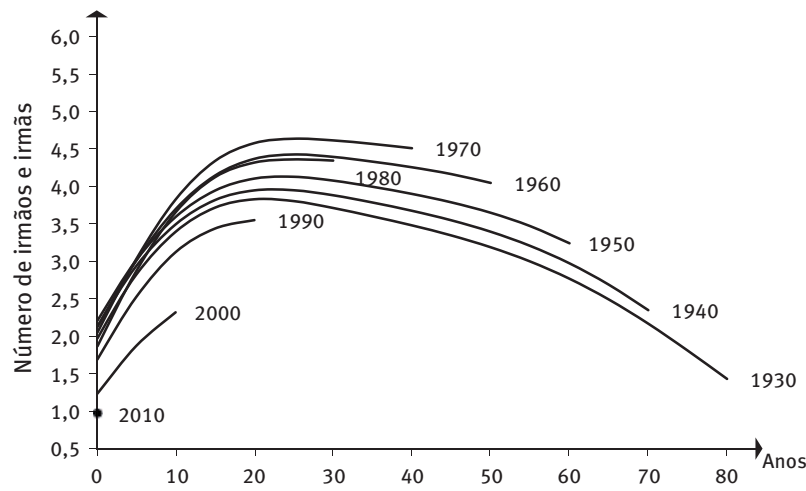

Fonte: Séries de taxas de fecundidade e mortalidade construídas por Horta, Carvalho e Frias (2000) e Wajnman (2012) baseadas em dados demográficos disponibilizados pelo IBGE.

Como era de se esperar, a proporção de irmãs sobre o número total de irmãs e irmãos é constante entre os nascidos vivos, mas cresce entre os sobreviventes, com o envelhecimento de ego, sem mostrar fortes evidências de mudanças neste padrão com a transição demográfica (Gráfico 2). Apesar disso, nota-se um ligeiro aumento na proporção de irmãs nas coortes intermediárias, provavelmente devido à ampliação do diferencial de mortalidade por sexo ocorrida especialmente entre 1980 e 2010, em função do crescimento das mortes por causas externas. 0 resultado dessas tendências é que, em suma, ego nasceria com quase tantas irmãs quanto irmãos, mas alcançaria os 80 anos com $20 \%$ mais irmãs do que irmãos. Considerando que irmãs tendem a desempenhar, com maior probabilidade, um papel mais ativo no suporte e cuidados com os idosos, essa evidência soma-se à do crescimento do número de irmãos (de ambos os sexos) sobreviventes para 
as coortes mais recentes de idosos, reforçando a ideia de que vivemos um momento demográfico excepcional, que não existiu no passado e nem se repetirá no futuro, em termos de disponibilidade de familiares na rede informal de suporte aos idosos.

GRÁFICO 2

Proporção de irmãs no número total de irmãs e irmãos sobreviventes, por coorte de nascimento de ego, segundo a idade de ego Brasil - 1930-2010

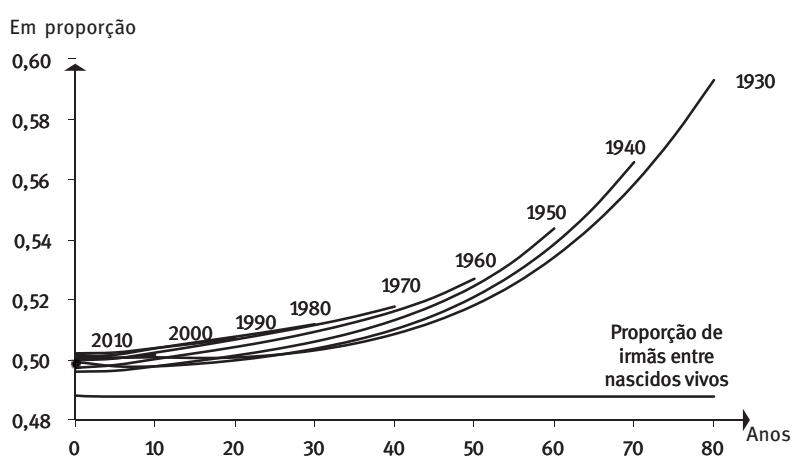

Fonte: Séries de taxas de fecundidade e mortalidade construídas por Horta, Carvalho e Frias (2000) e Wajnman (2012) baseadas em dados demográficos disponibilizados pelo IBGE.

0 Gráfico 3 exibe a evolução da proporção de irmãos mais novos sobre o número total de irmãos, nascidos vivos e estimados. Como se constata, até a idade de dez anos, aproximadamente, a proporção de irmãos mais novos aumenta, alcançando algo em torno de $50 \%$. Entre os nascidos vivos, esse percentual se mantém constante pelo resto da vida, mas, entre os sobreviventes, naturalmente, se eleva, atingindo algo em torno de $80 \%$, uma vez que a mortalidade age seletivamente entre os irmãos mais velhos. As alterações no nível das curvas observadas, entre as coortes de 1930 a 2010, acompanham a tendência de mudança da estrutura etária ocorrida ao longo dos estágios da transição demográfica brasileira, conforme observou Keyfitz (1986) e discutida na seção Antecedentes deste trabalho. Segundo essa tendência, taxas de crescimento populacional positivas elevadas são associadas a uma população mais jovem, na qual o percentual de irmãos mais jovens supera o de irmãos mais velhos, ao passo que taxas negativas (ou menos elevadas) associam-se a uma menor proporção de irmãos mais jovens. Note-se que, entre as coortes dos nascidos em 1960 e 1980, o percentual de irmãos mais jovens se eleva, para então começar a declinar, acompanhando a tendência de rejuvenescimento da estrutura etária e posterior início do processo de envelhecimento. 


\section{GRÁFICO 3}

Proporção de irmãs e irmãos mais novos no número total de irmãs e irmãos nascidos vivos e sobreviventes, por coorte de nascimento de ego, segundo a idade de ego Brasil - 1930-2010

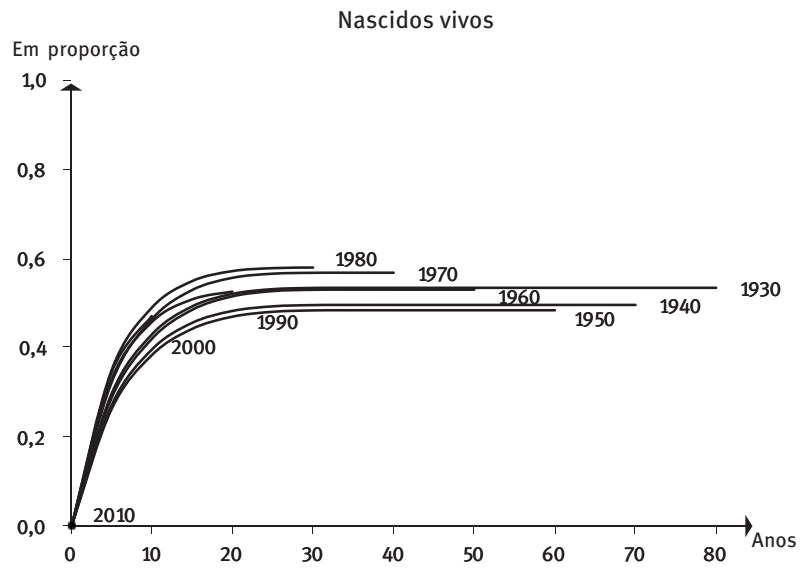

Sobreviventes

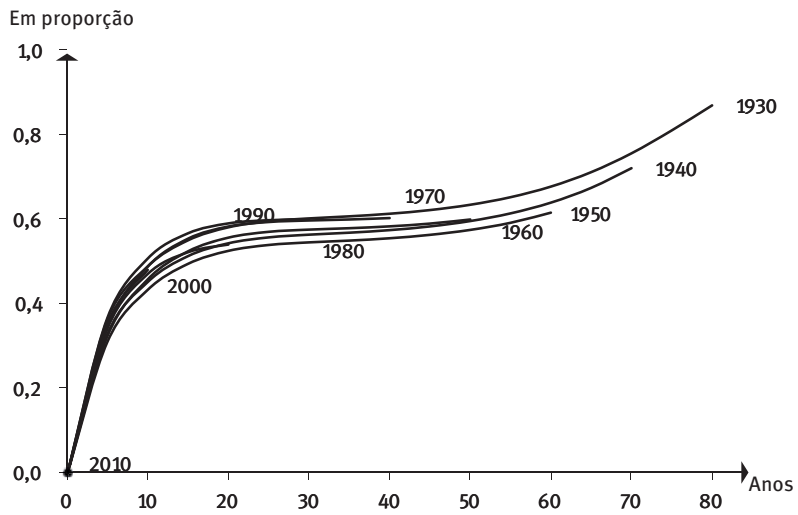

Fonte: Séries de taxas de fecundidade e mortalidade construídas por Horta, Carvalho e Frias (2000) e Wajnman (2012) baseadas em dados demográficos disponibilizados pelo IBGE.

Por fim, o Gráfico 4 mostra a evolução da proporção de mulheres entre egos (ou pessoas de referência) sobreviventes. Sendo ego uma pessoa aleatória de qualquer sexo, podemos identificar sua composição em termos dos percentuais de mulheres e homens sobreviventes em cada idade. Assim, como era de se esperar, à medida que envelhece, a proporção que compõe a pessoa média definida como ego se torna cada vez mais preponderantemente feminina, alcançando uma composição de $65 \%$ de mulheres aos 80 anos de idade. $\mathrm{Ou}$ seja, na perspectiva de pessoas de uma mesma geração, quando se examina a evolução do percentual de mulheres ao longo do ciclo de vida, constata-se uma proporção cada vez maior de mulheres, sejam elas as pessoas de referência (ego) ou suas irmãs. 
GRÁFICO 4

Proporção de mulheres entre egos sobreviventes, segundo a idade de ego

Brasil - 1930-2010

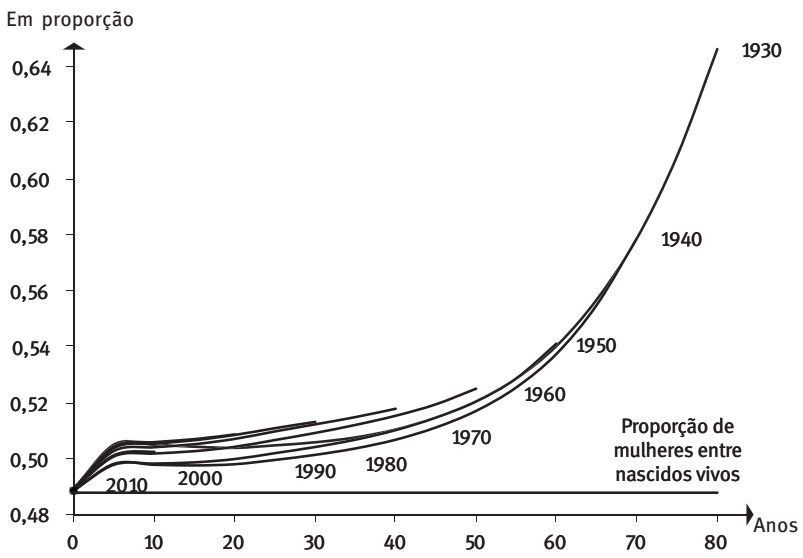

Fonte: Séries de taxas de mortalidade construídas por Horta, Carvalho e Frias (2000) e Wajnman (2012) baseadas em dados demográficos disponibilizados pelo IBGE.

\section{Discussão}

Inegavelmente, o método proposto por GKP configura-se como um avanço metodológico na demografia da família, ampliando o âmbito de investigação das relações de parentesco de dentro para fora do domicílio e permitindo o uso de informações indiretas, como funções de fecundidade e mortalidade, no lugar de pesquisas domiciliares para a construção do desenho das relações familiares. Ainda que essa metodologia esteja sujeita a restrições, algumas superáveis e outras não, sabemos que, invariavelmente, modelos matemáticos estão sujeitos a determinados pressupostos. Isso porque, ao considerarmos algumas condições como aceitáveis, podemos generalizar a modelagem a ser empregada e obter resultados plausíveis com a situação real. Por isso, ao escolhermos o método GKP, procuramos, primeiramente, contornar suas maiores limitações para, assim, estimar e interpretar apropriadamente os resultados.

Dessa forma, voltamos nossa atenção ao pressuposto de população estável, que é assumido no modelo proposto e nos trabalhos que, posteriormente, empregaram esta metodologia. Tal pressuposto tende a ser adotado por força da indisponibilidade de informações longitudinais sobre fecundidade e mortalidade e também pela praticidade, uma vez que, além de exigir poucos dados, simplifica os cálculos, por adotar, para todas as coortes, as mesmas funções vitais por idade. No entanto, o propósito deste trabalho era identificar, precisamente, o efeito da transição demográfica sobre a evolução do número de irmãos ao longo do tempo, com o que não faria sentido assumir funções vitais de fecundidade e mortalidade constantes entre as coortes de 1930 a 2010. Portanto, foram utilizadas uma longa série histórico-demográfica de taxas vitais e uma formulação mais complexa para 
a combinação das funções relativas a cada coorte nos sucessivos períodos, e produzidas estimativas de parentesco sob a perspectiva de coorte, gerando estimativas mais fiéis ao contexto transicional.

A segunda restrição do método GKP para a qual utilizamos uma alternativa refere-se à estimação de parentes limitada a somente o sexo feminino. Sem grandes dificuldades, esta tarefa mostrou-se ser a mais fácil e simples de enfrentar. Com pequenas adaptações nas fórmulas originais (por meio do emprego das taxas específicas de fecundidade de nascimentos masculinos e da sobrevivência masculina no caso dos irmãos, no lugar do uso das taxas específicas de fecundidade de nascimentos femininos e da sobrevivência feminina, no caso das irmãs, respectivamente), completamos as estimativas de irmãs e irmãos de ego, sem a exigência de dados mais detalhados.

Vale ressaltar que há, ainda, uma importante restrição da metodologia GKP que, neste trabalho, ainda não superamos: a estimação do número de parentes condicionada à fecundidade somente da mulher, que suscita a questão dos meios-irmãos paternos. Esta restrição não foi tratada devido à inexistência de dados que permitam superá-la. No caso brasileiro, as pesquisas domiciliares estabelecem as relações das pessoas residentes em um domicílio, definindo-as segundo sua relação com o responsável pelo domicílio. Caso o responsável e/ou seu cônjuge/companheiro(a) tenha(m) filhos corresidentes, estes serão relacionados como filhos (de um, de outro ou de ambos, dependendo da pesquisa), permitindo, assim, estabelecer somente o grupo de irmãos (incluindo-se irmãos biológicos, meios-irmãos e coirmãos) corresidentes desses filhos. Ocorre que, apesar de os filhos de antigas uniões permanecerem, de modo geral, com as mães, o mesmo não ocorre com os pais, de modo que os meios-irmãos paternos que vivem em domicílios diferentes tendem a ser invisíveis pelas informações domiciliares. Ao adotarmos um modelo de estimação que considera apenas a fecundidade feminina, do mesmo modo, perdemos de vista esse tipo de irmandade que, tudo indica, tende a ser cada vez mais comum, num cenário de recasamentos e filhos tidos com mais de um parceiro.

Finalmente, há um aspecto fundamental nos modelos analíticos de estimação de parentesco que deve ser bem compreendido: a questão da variabilidade populacional, que implica que as médias estimadas devem ser analisadas com cautela. No caso de nossas estimativas, há dois problemas. 0 primeiro diz respeito ao fato de que a fecundidade média da população incorpora a parturição zero, referente a mulheres que não tiveram filhos. No caso da estimativa do número de irmãos, no entanto, mulheres que não tiveram filhos não geram um ego para quem contaremos o número de irmãos e, portanto, não são computadas nas estimativas. Em outras palavras, é possível haver um número médio relativamente alto de irmãos, mesmo com a fecundidade média sendo muito baixa, caso haja uma elevada proporção de mulheres com parturição zero. Por isso, a estimativa mais adequada do número médio de irmãos deve levar em consideração não apenas a fecundidade média, mas também a sua distribuição. Esse problema foi endereçado em Guerra (2014), mas, dada a complexidade metodológica envolvida na incorporação de toda a distribuição 
da fecundidade, foi necessário assumir o pressuposto de estabilidade nas estimativas. Como neste trabalho estamos priorizando a análise da evolução do número de irmãos na transição demográfica, optamos por considerar, aqui, apenas a função do número médio de filhos tidos por idade. Desse modo, nossa estimativa do número médio de irmãos deve ser considerada subestimada, e não podemos tirar conclusões sobre a distribuição do número de irmãos na população.

O outro problema refere-se precisamente à distribuição do número de irmãos na população. 0 modelo de estimativa adotado utiliza funções médias de fecundidade e mortalidade e não considera nem a heterogeneidade populacional, ${ }^{1}$ nem a interdependência entre fecundidade e mortalidade. Ruggles (1993) alerta que o problema causado pela suposição de independência na estimação de parentesco nos modelos KGP relaciona-se tanto com a heterogeneidade de eventos demográficos na população quanto com a homogeneidade observada dentro dos grupos familiares. Coresh e Goldman (1988) também discutem o problema da suposição implícita de homogeneidade das funções de fecundidade e mortalidade nos modelos KGP e propõem uma modificação dos métodos de estimação, a fim de incorporar a heterogeneidade da fecundidade na análise dos filhos e dos irmãos. Apesar de permitir variabilidade na fecundidade por subgrupos da população, os autores assumem fecundidade e mortalidade fixas ao longo do tempo. Por meio de macrossimulações, eles demonstram que, como esperado, o número médio de irmãos nascidos vivos e sobreviventes para todas as idades de ego é subestimado ao se considerar a fecundidade populacional homogênea. ${ }^{2}$

\section{Conclusão}

Ao início da transição demográfica, a média de irmãos nascidos vivos se encontra em patamares elevados e, como esperado, sofre um declínio durante o processo. Nossos resultados mostram que o número de irmãos sobreviventes nas idades mais avançadas tende a ser muito semelhante para coortes mais velhas e mais novas. No entanto, o número de irmãos sobreviventes durante a infância dessas coortes tende a diferenciar-se imensamente. Isso porque, por um lado, o efeito da alta mortalidade, principalmente a infantil, reduz a média de irmãos gerados pela alta fecundidade para as coortes mais velhas e, por outro lado, a queda da fecundidade diminui o número de irmãos nascidos vivos das coortes mais

\footnotetext{
${ }^{1}$ Cavenaghi e Goldani (1993), analisando os dados censitários brasileiros, identificam a diferença nos tamanhos das famílias sob a perspectiva das mulheres, em termos de número médio de filhos, e sob a perspectiva das crianças, em termos de número médio de irmãos. Para mensurar o tamanho da família das mulheres, as autoras propõem o cálculo da descendência média final e observam que tanto a descendência média final como o número médio de irmãos apresentam diferentes padrões quando se toma a distribuição das crianças por região de residência, cor e nível de instrução da mãe e coorte de origem das mães.

${ }^{2}$ Cabe esclarecer que, embora as reformulações sugeridas por Coresh e Goldman (1988) considerem a existência de heterogeneidade da fecundidade, a heterogeneidade da mortalidade não foi incorporada nos métodos apresentados, o que deixa uma lacuna a ser preenchida na questão de como as heterogeneidades da fecundidade e da mortalidade podem afetar, simultaneamente, as relações de parentesco e, consequentemente, a dinâmica demográfica em uma população real.
} 
novas, ao mesmo tempo que a queda da mortalidade permite que não haja decrementos significativos ao longo do ciclo de vida.

Mesmo que a disponibilidade de irmãs e irmãos não tenha sido construída para todo o ciclo de vida das coortes estudadas, é possível perceber duas importantes tendências, como resultado da transição demográfica: o número médio de irmãos nascidos vivos tende a se estabelecer em baixos níveis nos próximos anos, motivado principalmente pela queda da fecundidade, ao mesmo tempo que o número médio de irmãos sobreviventes tende a ser cada vez mais próximo do número médio de irmãos nascidos vivos, devido, principalmente, à queda da mortalidade. Outros achados importantes referem-se ao fato de que a proporção de irmãs tende a crescer ao longo do ciclo de vida, por efeito da mortalidade diferencial por sexo, mas também se elevou entre as coortes de 1930 e as nascidas em períodos mais recentes, possivelmente pela atuação da mortalidade por causas externas, que acomete mais os homens. Junto a isso, a proporção de irmãos (ambos os sexos) mais novos sobreviventes tende a se elevar no ciclo de vida, pela ação da mortalidade, e aumentou particularmente para as coortes que nasceram sob o regime de elevadas taxas de natalidade. Combinadas estas tendências, concluímos que o cenário demográfico atual favorece uma oferta de irmãos sobreviventes para os idosos atuais maior do que se tinha no passado e que se terá no futuro, o que tende a se constituir em um importante elemento na formação da rede de suporte informal aos idosos, sobretudo no contexto de maior instabilidade dos casamentos e menor propensão dos filhos a se manterem geograficamente próximos de seus pais.

Por fim, vale chamar a atenção para o fato de que, de acordo com a taxa de fecundidade total (TFT) de 1,8 filho por mulher observada em 2010 pelo IBGE, poderíamos admitir que a moda esperada da parturição para esse nível de fecundidade estaria entre um e dois filhos por mulher. Isso poderia provocar a impressão de que, nas atuais condições da transição demográfica brasileira, seria esperada a média de 0,8 irmão por filho e a moda esperada estaria entre zero e um irmão por filho. No entanto, conforme discutido na seção anterior, o nível da fecundidade média não permite a inferência direta do número médio de irmãos, uma vez que o número de irmãos depende da proporção de mulheres que não têm filhos. Com base na TFT atual, podemos admitir uma representativa proporção de filhos únicos na população como uma tendência demográfica atual. Por outro lado, a distribuição das mulheres brasileiras por parturição mostra que cerca de 3 em cada 4 mulheres com filho(s) em 2010, em idade reprodutiva, têm pelo menos dois filhos, o que corrobora o ponto que enfatizamos quanto à questão da distribuição da fecundidade, indicando não ser desprezível a presença de irmãos nas famílias que têm filhos. Assim, com base na hipótese de manutenção do nível aproximado da fecundidade corrente, podemos admitir que, embora ser filho único tenha se tornado comum nos dias atuais, a extinção dos irmãos e da colateralidade no parentesco não pode ser considerada uma tendência no Brasil.

Como agenda de trabalhos futuros, sobressai a necessidade de incorporarmos a heterogeneidade populacional, sobretudo a heterogeneidade socioeconômica, para 
uma estimativa da distribuição do número de irmãos. As consequências da distribuição de irmãos segundo características socioeconômicas têm implicações evidentes sobre as perspectivas diferenciadas das necessidades de cuidados de idosos e sobre a transmissão intergeracional da riqueza, por meio das heranças, para citar apenas alguns exemplos de aplicação desse tipo de estudo.

\section{Referências}

BIGBY, C. Parental substitutes: the role of siblings in the lives o folder people with intelectual disability. Journal of Gerontological Social Work, v. 29, n. 1, 1998.

BURCH, T. K. Estimating the Goodman, Keyfitz, Pullum kinship equations: an alternative procedure. Mathematical Population Studies, v. 5, n. 2, p. 161-170, 1995.

CAVENAGHI, S. M.; GOLDANI, A. M. Fecundidade e família: os tamanhos das famílias das mulheres e das crianças no Brasil. Revista Brasileira de Estudos de População, v. 10, n. 1, p. 107-124, 1993.

CORESH, J.; GOLDMAN, N. The effect of variability in the fertility schedule on numbers of kin. Mathematical Population Studies, v. 1, n. 2, p. 137-156, 1988.

ERIKSEN, S.; GERSTEL, N. A labor or love or labor itself: care work among adult brothers and sisters. Journal of Family Issues, v. 23, n. 7, p. 836-856, 2002.

GOLDMAN, N. Estimating the intrinsic rate of increase of a population from the average numbers of younger and older sisters. Demography, v. 14, n. 4, p. 499-507, 1978.

GOODMAN, L.; KEYFITZ, N.; PULLUM, T. W. Addendum. Family formation and the frequency of various kinship relationships. Theoritical Population Biology, v. 8, p. 376- 381, 1975.

Family formation and the frequency of various kinship relationships. Theoritical Population Biology, v. 5, p. 1-27, 1974.

GRIFFITH, J. D.; KOO, H. P. Childbearing and family in remarriage. Demography, v. 22, n. 1, p. $73-88,1985$.

GUERRA, F. F. Transição e tendências da disponibilidade de irmãos para o Brasil: um estudo metodológico sobre relações de parentesco. 106f. Tese (Doutorado) - Departamento de Demografia, Faculdade de Ciências Econômicas, Universidade Federal de Minas Gerais, Belo Horizonte, 2014.

HAMMEL, E. A. Demographic dynamics and kinship in anthropological populations. PNAS, v. 102, n. 6, p. 2248-2253, 2005 a.

Kinship-based politics and the optimal size of kin groups. PNAS, v.102, n. 33, p. 11951-11956, 2005b.

HORTA, C. J.; CARVALHO, J. A. M.; FRIAS, L. A. M. Recomposição da fecundidade por geração para Brasil e regiões: atualização e revisão. In: ENCONTRO NACIONAL DE ESTUDOS POPULACIONAIS, XII., 2000. Anais... Caxambu-MG: Abep, 2000. p. 1-22.

KEISTER, L. A. Sharing the wealth: the effect of siblings on adults' wealth ownership. Demography, v. 40, n. 3, p. 521-542, 2003.

KEYFITZ, N. Canadian kinship patterns based on 1971 and 1981 data. Canadian Studies in Population, v. 13, n. 2, p.123-150, 1986. 
KEYFITZ, N.; CASWELL, H. Applied mathematical demography: statistics for biology and health. 3. ed. New York: Springer, 2005.

MANTON, K. G.; STALLARD, E.; VAUPEL, J. W. The impact of heterogeneity in individual frailty on the dynamics of mortality. Demography, v. 16, n. 3, p. 439-454, 1979.

MCDANIEL, C. K.; HAMMEL, E. A. A kin-based measure of $r$ and an evaluation of its effectiveness. Demography, v. 21, n. 1, p. 41-51, 1984.

MURPHY, M. Changes in family and kinship networks consequent on the demographic transitions in England and Wales. Continuity and Change, v. 25, p. 109-136, 2010.

. Long-term effects of the demographic transition on cohorts' demographic experiences in Britain. In: WORKSHOP LONG TERM IMPLICATIONS OFTHE DEMOGRAPHIC TRANSITION. Madri, 2009.

PRESTON, S. H.; HEUVELINE, P.; GUILLOT, M. Demography: measuring and modeling population processes. Oxford: Blackwell Publishers, 2001.

PULLUM, T. W. The eventual frequencies of kin in a stable population. Demography, v. 19, n. 4, p. 549-565, 1982.

PULLUM, T. W.; WOLF, D. A. Correlations between frequencies of kin. Demography, v. 28, n. 3, p. 391-409, 1991.

THOMSON, E. Her, his and their children: Influences on couple childbearing decisions. WisconsinMadison: A National Survey of Families and Households, 1997 (NSFH working paper, n. 89).

Step-families and childbearing desires in Europe. Demographic Research, Coleção especial n. 3, artigo 5, p.117-134, 2004.

VIKAT, A.; THOMSON, E.; HOEM, J. M. Stepfamily fertility in contemporary Sweden: the impact of childbearing before the current union. Population Studies, n. 3, p. 211-225, 1999.

WACHTER, K. W. The sisters' riddle and the importance of variance when guessing demographic rates from kin counts. Demography, v. 17, n. 1, p. 103-114, 1980.

WAJNMAN, S. Demografia das famílias e dos domicílios brasileiros. 161f. Tese (Professor Titular) - Departamento de Demografia, Faculdade de Ciências Econômicas, Universidade Federal de Minas Gerais, Belo Horizonte, 2012.

WHITE, L. Sibling relationships over the life course: a panel analysis. Journal of Marriage and Family, v. 63, p. 555-568, 2001.

WOLF, D.; FREEDMAN, V.; SOLDO, B. The division of family labor: care of elderly parents. The Journals of Gerontology Series, v. 52.B, p.102-109, 1997.

\section{Sobre os autores}

Francismara Fernandes Guerra é doutora em Demografia pelo Cedeplar, UFMG. Atualmente é pós-doutoranda no Departamento de Economia Doméstica da Universidade Federal de Viçosa.

Simone Wajnman é doutora em Demografia pelo Cedeplar, UFMG. Professora no Centro de Desenvolvimento e Planejamento Regional (Cedeplar), da Universidade Federal de Minas Gerais (UFMG).

Cássio M. Turra é doutor em Demografia pela Universidade da Pensilvânia, Estados Unidos. Professor no Centro de Desenvolvimento e Planejamento Regional (Cedeplar), da Universidade Federal de Minas Gerais (UFMG). 


\title{
Endereço para correspondência
}

\author{
Francismara Fernandes Guerra \\ Departamento de Economia Doméstica, Universidade Federal de Viçosa \\ Av. Peter Henry Rolfs s/ $\mathrm{n}$ - campus universitário \\ 36570-000 - Viçosa-MG, Brasil
}

Simone Wajnman

Faculdade de Ciências Econômicas, Departamento de Demografia, Universidade Federal de Minas Gerais

Av. Antônio Carlos, 6.627 - campus Pampulha

31270-901 - Belo Horizonte-MG, Brasil

Cássio M. Turra

Faculdade de Ciências Econômicas, Departamento de Demografia, Universidade Federal de Minas Gerais

Av. Antônio Carlos, 6.627 - campus Pampulha

31270-901 - Belo Horizonte-MG, Brasil

\begin{abstract}
Availability of siblings in Brazil: a methodological study on kinship relationships

When fertility declines, it is not only the number of children that becomes smaller, but the number of siblings as well. To determine changes in the number of siblings over time in Brazil, this study uses a method that is designed to estimate, through mathematical models which use only fertility and mortality rates, the availability of surviving siblings in different cohorts. The results indicate that, at the beginning of the demographic transition, the mean number of the born alive siblings is established at high levels and suffers a sharp decline during the transition, mainly due to falling fertility. They also show that the mean number of the surviving siblings at older ages tends to be very similar for older and younger cohorts. However, the mean number of surviving siblings during the childhood of these cohorts tends to differ greatly. This is due to high mortality, especially infant mortality, on the one hand and, on the other, from fertility decline which reduces the number of live births in more recent cohorts while the reduction of mortality increases their chances of survival. The study's conclusion points out the following trends: the mean number of surviving siblings will tend to settle at lower levels in coming years and the mean number of surviving siblings tends to be increasingly closer to the mean number born alive. Despite current low levels of fertility, it would be incorrect to speak of the extinction of siblings and, consequently, of cousins, uncles, etc.
\end{abstract}

Keywords: Demographic transition. Kinship models. Availability of siblings.

\section{Resumen}

Disponibilidad de hermanos en Brasil: un estudio metodológico sobre las relaciones de parentesco

La reducción de la fecundidad reduce no sólo solamente el número de niños, pero también el número de hermanos. Para evaluar los cambios en la disponibilidad de hermanos en Brasil, se utilizó un método que estima el número esperado de hermanos nacidos vivos y sobrevivientes 
por medio de modelos matemáticos que utilizan solamente las tasas de fecundidad y mortalidad. Nuestros resultados indican que, en el comienzo de la transición demográfica, el promedio de hermanos nacidos vivos se establece en niveles altos y sufre una fuerte caída durante la transición, debido principalmente a la caída de la fecundidad. Los resultados muestran que el promedio de hermanos sobrevivientes a edades más avanzadas tiende a ser muy similar para las cohortes de más edad y las más jóvenes. Sin embargo, el número medio de hermanos sobrevivientes durante la infancia de estas cohortes tiende a variar mucho. Esto ocurre por dos razones. Por un lado, la mortalidad elevada, principalmente la infantil, reduce el número medio de hermanos para las cohortes más viejas. Por otro, el descenso de la fecundidad disminuye el número de nascidos vivos en las cohortes más nuevas, mientras la reducción de la mortalidad aumenta sus posibilidades de sobrevivencia. En conclusión, las siguientes tendencias son señaladas: el número promedio de hermanos nacidos vivos tiende a establecerse en niveles bajos en los próximos años, el número medio de hermanos sobrevivientes tiende a ser más cerca de la media de nacidos vivos, y a pesar de la reducción de la fecundidad, no hay evidencia de una posible extinción de los hermanos y, en consecuencia, de primos, tíos, etc.

Palabras clave: Transición demográfica. Estimación del parentesco. Disponibilidad de hermanos.

Recebido para publicação em 04/08/2015 Recomendado para publicação em 23/03/2016

Aceito para publicação em 21/04/2016 
